\title{
Expression of histone deacetylase 1 and metastasis-associated protein 1 as prognostic factors in colon cancer
}

\author{
JUN HIGASHIJIMA, NOBUHIRO KURITA, TOMOHIKO MIYATANI, KOZO YOSHIKAWA, \\ SHINYA MORIMOTO, MASANORI NISHIOKA, TAKASHI IWATA and MITSUO SHIMADA \\ Department of Surgery, Institute of Health Biosciences, The University of Tokushima, Tokushima 770-8503, Japan
}

Received January 21, 2011; Accepted April 4, 2011

DOI: $10.3892 /$ or.2011.1312

\begin{abstract}
Histone deacetylase 1 (HDAC1) and metastasisassociated protein 1 (MTA1) form the nucleosome remodeling and histone deacetylation (NuRD) complex and may possibly play a central role in cancer development. However, limited data has been reported regarding the expression of both HDAC1 and MTA1. The aim of the present study was to clarify the clinical role of HDAC1 and MTA1 expression in colon cancer. Seventy-four patients with colon cancer, who underwent colectomy at our institution, were enrolled in this study. Expression of HDAC1 and MTA1 was examined immunohistochemically. The patients were divided into four groups: HDAC1-positive group ( $\mathrm{n}=58$ ), HDAC1-negative group $(n=16)$, MTA1-positive group $(n=38)$ and MTA1-negative group $(n=36)$. Clinicopathological factors and survival rates were compared between the groups. Regarding the clinicopathological factors, the depth of tumor invasion and stage correlated significantly with HDAC1 expression $(\mathrm{p}<0.05)$. Age, depth of tumor invasion and vascular invasion tended to correlate with MTA1 expression. The 5-year survival rate in the HDAC1-positive group (55.1\%) was significantly worse compared to the HDAC1-negative group $(86.5 \%)(\mathrm{p}<0.05)$, and the 5-year survival rate of the MTA1-positive group (50.5\%) was significantly worse than that of the MTA1-negative group $(73.1 \%)(\mathrm{p}=0.05)$. In patients with stages II-IV and curability A, B, the survival rate in those with HDAC1-positive expression was significantly worse than those with HDAC1negative expression $(\mathrm{p}<0.05)$, and the survival rate of the MTA1-positive group tended to be worse than that of the MTA1-negative group ( $\mathrm{p}=0.07)$. Overall survival in both the HDAC1 and MTA1-positive groups was significantly worse than overall survival of the other groups $(\mathrm{p}<0.05)$. Disease-free
\end{abstract}

Correspondence to: Dr Nobuhiro Kurita, Department of Surgery, The University of Tokushima, 3-18-15 Kuramoto-cho, Tokushima 770-8503, Japan

E-mail: kurita@clin.med.tokushima-u.ac.jp

Key words: histone deacetylase 1, metastasis-associated protein 1, advanced colon cancer, epigenetic alteration, immunohistochemistry, survival rate, disease-free survival rate survival in both the HDAC1- and MTA1-positive groups, among patients with stages II-IV and curability A, B, was also significantly worse than that of the other groups $(p<0.05)$. HDAC1 and MTA1 expression levels were significantly related to poorer prognosis. Therefore, HDAC1 and MTA1 expression levels are potential prognostic indicators for colon cancer.

\section{Introduction}

Colorectal cancer is one of the major causes of cancer-related mortality in the world $(1,2)$. This disease is surgically curable at early stages, but is associated with high mortality rates at the metastatic stage. Although advances in the chemotherapeutic regimens and combination with radiotherapy have markedly improved the survival of patients with advanced colorectal cancer (3), surgery is the only curative form of treatment. Moreover, the current therapeutic concepts, particularly for late-stage tumors, are far from optimal. Thus, even though mortality rates have improved, new prognostic biomarkers and therapeutic targets are still urgently needed for colorectal cancer.

Epigenetic modifications including alterations of the acetylation status of histones play a central role in the development of human cancer (4). Acetylation and deacetylation on different positions of the N-terminal tail of core histones by histone acetylases and histone deacetylases (HDACs) change the nucleosomal conformation of both transformed and nontransformed cells (5).

To date, 18 HDAC isoforms have been identified which are classified into four classes based upon their homology to a prototypical HDAC found in yeast (5): class I isoforms $(1-3,6)$, class II isoforms $(4,5,7,8)$, class III isoforms (the sirtuins) and class IV isoforms (HDAC11).

Class I HDACs play an important role in the regulation of cell proliferation (6), and overexpression of class I HDACs have been reported in several types of cancer tissues, such as esophagus (7), stomach $(8-10)$, pancreas $(11)$, prostate $(12,13)$, breast $(14,15)$ and lung $(16,17)$.

HDACs are the core components of the nucleosome remodeling and histone deacetylation (NuRD) complex, and most of the components of this complex have been related to malignancy $(18,19)$. In addition, metastasis-associated gene 1 (MTA1) protein is also included in this complex and physically interacts with HDAC1 (19). This protein complex plays an 


\section{HDAC1}

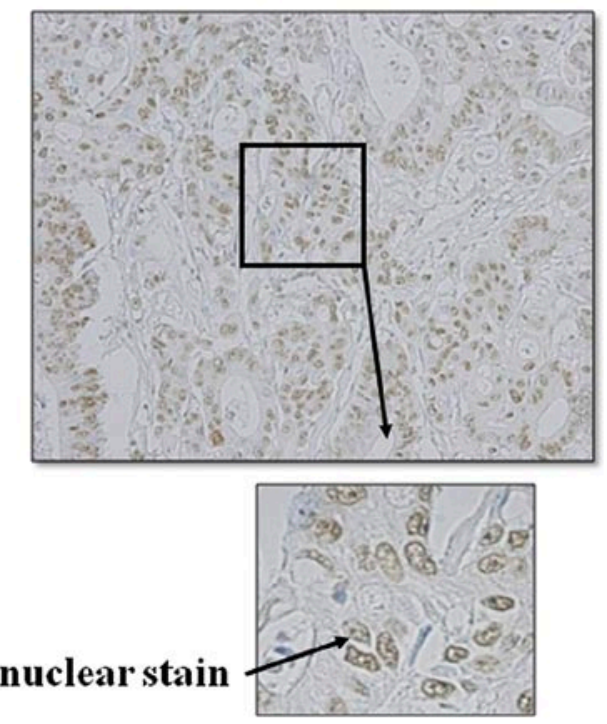

\section{MTA1}

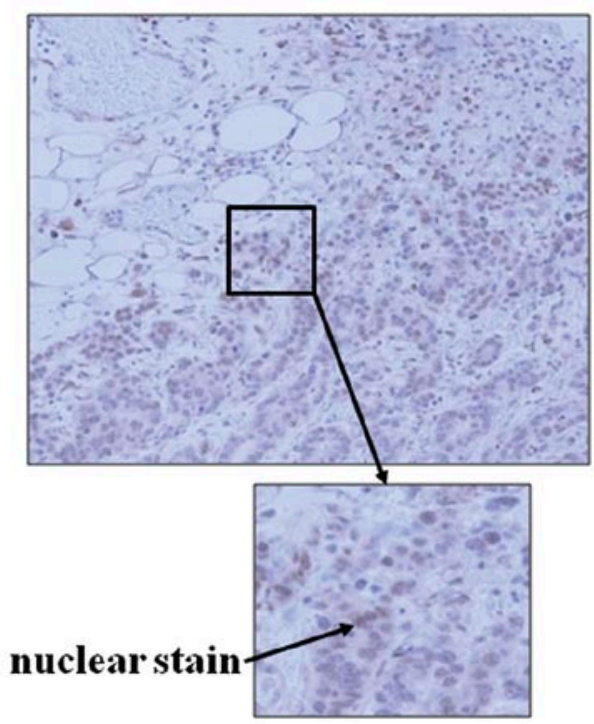

Figure 1. HDAC1 and MTA1 expression in colon cancer. Nuclear staining of HDAC1 and MTA1 was recognized in the tumor tissue.

important role in histone deacetylation, alteration of chromatin structure and transcriptional control.

MTA is a newly discovered family of cancer progressionrelated genes, and the MTA family is a group of structurally related proteins encoded by the same or different genes, including MTA1, MTA1s, MTA-ZG29p, MTA2, MTA3 and MTA3L $(21,22)$.

Increased expression of MTA1 is reported in various types of human cancer tissues, such as esophagus $(22,23)$, stomach (24), pancreas (24), prostate (25), breast $(26,27)$ and lung (28). However, little has been reported regarding the correlation between prognosis and expression of both HDAC1 and MTA1 in colon cancer. This study, therefore, aimed to identify the expression pattern of HDAC1 and MTA1 in colon cancer patients, and the correlation with clinicopathological data and patient survival.

\section{Materials and methods}

Patients. The study groups consisted of 74 colon cancer patients who had undergone surgery at our institution between 2000 and 2004. The colon tumors were classified according to the General Rule for Clinical and Pathological Studies on Cancer of the Colon, Rectum and Anus in Japan (29). The clinical and pathological characteristics of the 74 colon cancer patients are listed in Table I. Curability was defined as follows: curability A, no residual tumor; curability B, no residual tumor but not evaluated as curability $\mathrm{A}$; and curability $\mathrm{C}$, definite residual tumors. This study was authorized in advanced by the Institutional Review Board of the University of Tokushima Graduate School, and all patients provided written informed consent.

\section{Immunohistochemistry}

HDAC1. Surgical specimens were fixed in $10 \%$ formaldehyde, embedded in paraffin and cut into $4-\mu \mathrm{m}$ sections. Sections were deparaffinized in xylene and rehydrated in a graded series of ethanol. Slides were heated at $120^{\circ} \mathrm{C}$ in an autoclave in $10 \mathrm{mM}$ sodium citrate $(\mathrm{pH} 6.0$ ) for $10 \mathrm{~min}$ and cooled to room temperature. Sections were incubated in $30 \%$ hydrogen peroxide in methanol for 20 min to block endogenous peroxidase action. Sections were incubated overnight at $4^{\circ} \mathrm{C}$ with rabbit polyclonal antibody against HDAC1 (Santa Cruz, sc-7872; diluted 1:100 in PBS). After washing, sections were overlaid with secondary antibody (Dako ChemMate Envision kit/HRP; Dako Corporation) for $60 \mathrm{~min}$ at $37^{\circ} \mathrm{C}$. The peroxidase reaction was developed with 3,3'-diaminobenzidine as chromogen. The sections were counterstained with hematoxylin, dehydrated with ethanol, treated with xylene and enclosed in synthetic resin. Regarding the assessment of staining, the tumor was defined as exhibiting positive staining when $>10 \%$ nuclear staining of HDAC1 was noted in the tumor tissue.

MTA1. Sections (4- $\mu \mathrm{m})$ were cut from archival formalin-fixed paraffin-embedded tissue blocks. Sections were then irradiated in a domestic microwave oven for $20 \mathrm{~min}$. After microwave irradiation, the slides were allowed to cool at room temperature. The sections required a primary mouse monoclonal antibody against MTA1 (Santa Cruz, sc-17773; diluted 1:10 in PBS). Regarding the assessment of staining, the tumor was defined as exhibiting positive staining when $>10 \%$ nuclear staining of HDAC1 was noted in the tumor tissue. Representative images of nuclear staining of HDAC1 and MTA1 in tumor tissues are shown in Fig. 1.

Statistical analysis. To compare continuous variables, the Mann-Whitney U test was used, and the $\chi^{2}$ test was applied for categorical data. Patient survival was calculated by the product limit method of Kaplan and Meier, and differences in survival rates between the groups were compared using the log-rank test. The continuous variables were generally classified into two groups, according to the median value of each variable. All statistical analysis was performed using StatView-J 5.0 software (SAS Institute, Cary, NC, USA). $\mathrm{p}<0.05$ was considered statistically significant. 
Table I. HDAC1 expression and clinicopathological data of 74 colon cancer patients.

\begin{tabular}{lcc}
\hline & \multicolumn{2}{c}{ HDAC1 expression } \\
\cline { 2 - 3 } Factors & Positive (n=58) & Negative (n=16) \\
\hline Age: median (range) & $67.0(26-89)$ & $68.4(54-87)$ \\
Gender: (male/female) & $34 / 24$ & $11 / 5$ \\
Depth of tumor invasion: (T2/T3/T4) & $21 / 31 / 6$ & $14 / 2 / 0$ \\
Lymph node metastasis: (+/-) & $33 / 25$ & $6 / 10$ \\
Vascular invasion: (+/-) & $27 / 31$ & $6 / 10$ \\
Distant metastasis: (+/-) & $24 / 34$ & $3 / 13$ \\
Stage: (I/II/III/IV) & $2 / 12 / 20 / 24$ & $2 / 8 / 3 / 3$ \\
Differentiation: (differentiated vs. undifferentiated) & $54 / 4$ & $16 / 0$ \\
Curability: (A, B/C) & $38 / 20$ & 0.001 \\
\end{tabular}

Table II. MTA1 expression and clinicopathological data of 74 colon cancer patients.

\begin{tabular}{lcc}
\hline & \multicolumn{2}{c}{ MTA1 expression } \\
\cline { 2 - 3 } Factors & Positive (n=38) & Negative (n=36) \\
\hline Age: median (range) & $65.2(39-84)$ & $69.5(26-89)$ \\
Gender: (male/female) & $25 / 13$ & $20 / 16$ \\
Depth of tumor invasion: (T2/T3/T4) & $14 / 21 / 3$ & $21 / 12 / 3$ \\
Lymph node metastasis: (+/-) & $22 / 16$ & $17 / 19$ \\
Vascular invasion: (+/-) & $21 / 17$ & $12 / 24$ \\
Distant metastasis: (+/-) & $15 / 23$ & $12 / 24$ \\
Stage: (I/II/III/IV) & $2 / 10 / 11 / 15$ & 0.14 \\
Differentiation: (differentiated vs. undifferentiated) & $37 / 1$ & 0.15 \\
Curability: (A, B/C) & $25 / 13$ & $33 / 3$ \\
\hline
\end{tabular}

A

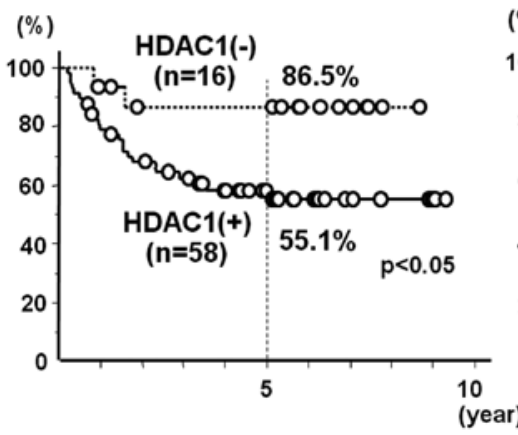

B

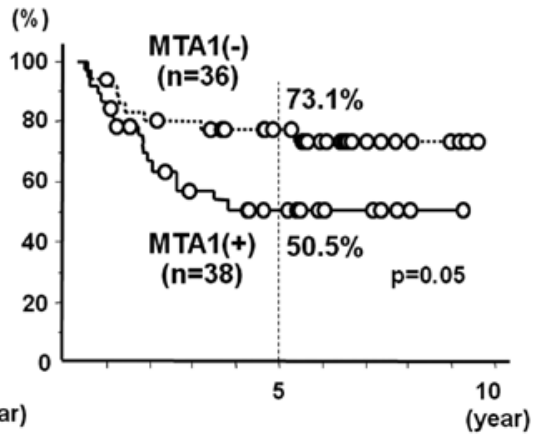

Figure 2. Overall survival according to HDAC1 (A) and MTA1 (B) expression patterns. The survival rates in the HDAC1- and MTA1-positive groups were significantly worse than that of the HDAC1- and MTA1-negative groups.

\section{Results}

Table I shows a comparison of the clinicopathological features between the HDAC1-positive and HDAC1-negative groups. The depth of tumor invasion and stage significantly correlated with HDAC1 expression ( $\mathrm{p}<0.05)$, and distant metastasis, curability and differentiation tended to correlate with HDAC1 expression.

Table II shows a comparison of the clinicopathological features between the MTA1-positive and MTA1-negative groups. Age, depth of tumor invasion and vascular invasion tended to correlate with MTA1 expression.

Fig. 2 shows the comparison of the overall survival curves according to the status of HDAC1 and MTA1 expression. The 5-year survival rate in the HDAC1-positive group (55.1\%) was significantly worse than that of the HDAC1-negative group $(86.5 \%)(\mathrm{p}<0.05)($ Fig. 2A). The 5-year survival rate in the MTA1-positive group (50.5\%) was significantly worse than that of the MTA1-negative group (73.1\%) $(\mathrm{p}=0.05)$ (Fig. 2B). 


\section{A}

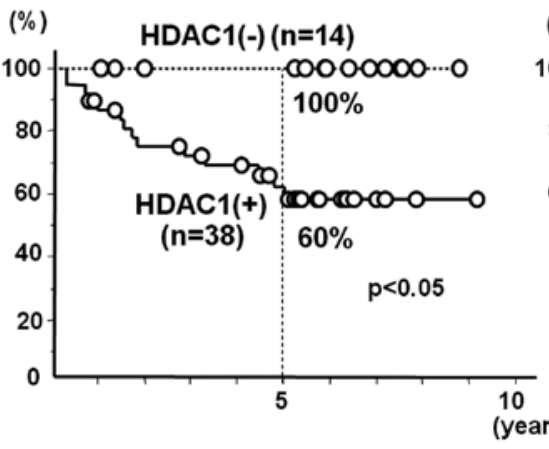

B

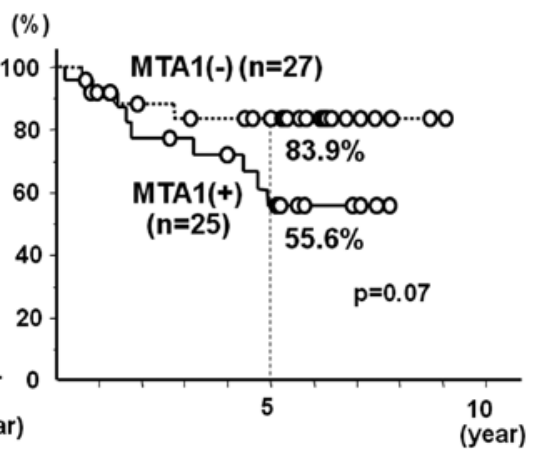

Figure 3. Disease-free survival according to (A) HDAC1 and (B) MTA1 expression patterns in stages II-IV and curability A, B patients. The survival rate in the HDAC1-positive group was significantly worse than that of the HDAC1-negative group, and the survival rate of the MTA1-positive group tended to be worse than that of the MTA1-negative group.

A

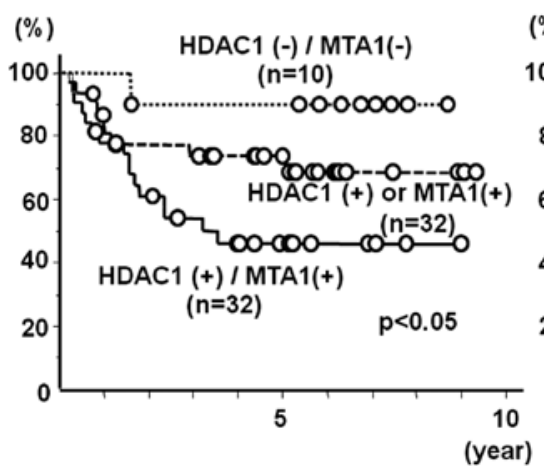

B

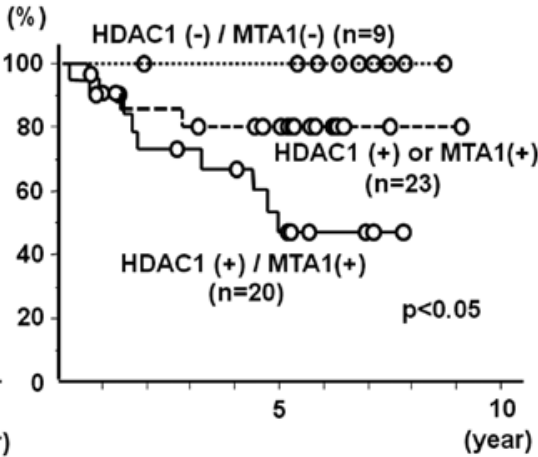

Figure 4. Overall and disease-free survival according to one or both of patterns of HDAC1 and MTA1 expression. (A) Overall survival according to one or both HDAC1 and MTA1 expression patterns. (B) Disease-free survival according to one or both HDAC1 and MTA1 expression patterns in stages II-IV and curability A, B patients. The survival rates in the HDAC1- and MTA1-positive groups were significantly worse than the rates in the HDAC1- and MTA1negative groups.

Fig. 3 shows a comparison of the disease-free survival curves according to the status of HDAC1 and MTA1 expression in stages II-IV and curability A, B patients. The survival rate in the HDAC1-positive group was significantly worse than that of the HDAC1-negative group ( $\mathrm{p}<0.05)(\mathrm{Fig} 3 \mathrm{~A})$. The survival rate of the MTA1-positive group tended to be worse than that of the MTA1-negative group ( $\mathrm{p}=0.07$ ) (Fig. 3B).

Fig. 4 shows a comparison of the overall survival and disease-free survival curves according to the status of HDAC1 and MTA1 expression. Overall survival in the HDAC1- and MTA1-positive groups was significantly worse than that of the other groups $(\mathrm{p}<0.05)$ (Fig. 4A). Disease-free survival in the HDAC1- and MTA1-positive groups among the patients with stages II-IV and a curability A, B was also significantly worse than that of the other groups $(\mathrm{p}<0.05)$ (Fig. 4B).

\section{Discussion}

In the present study, the findings are as follows: i) HDAC1 expression was significantly related to histological tumor status and stage; ii) MTA1 expression tended to be related to tumor status and vascular invasion; and iii) HDAC1 and
MTA1 expression was associated with decreased patient survival in colon cancer.

Regarding the relationship between clinicopathological factors and HDAC1 or MTA1, HDAC1 mRNA levels were reported to be significantly associated with tumor stage and HDAC1 protein expression in lung cancer (16), and MTA1 expression was reported to be significantly associated with depth of tumor invasion, lymph node metastasis, tumor stage, and lymphatic and vascular invasion in esophageal squamous cell carcinomas (22). These reports support our finding that HDAC1 and MTA1 expression was significantly related with various clinicopathological factors in colon cancer.

Regarding the prognostic value of HDAC1 and MTA1, in gastric cancer, HDAC1 expression was significantly found to be related to poor prognosis (9). In colon cancer, Weichert et al (30) reported that the overall survival rate in HDAC1positive patients was significantly lower than the survival rate in HDAC1-negative patients. Toh et al (22) reported that the overall survival rate of MTA1-positive patients with esophageal cancer was significantly lower than the rate in MTA1-negative patients. Miyake et al (31) reported that the expression level of MTA1 protein in combination with the 
expression level of HDAC1 protein correlated with a poorer prognosis of pancreatic cancer patients. These reports support our finding that levels of HDAC1 and MTA1 expression are important prognostic indicators in colon cancer.

HDACs and HDAC inhibitors (HDACi) have important roles in colon cancer. Mariadason reported in his review (32) that HDAC overexpression may facilitate the progression of colon tumors through two mechanisms. First, HDAC overexpression can contribute to the transcriptional repression of genes that normally function in growth arrest, differentiation and apoptosis, by inducing histone hypoacetylation in core promoter regions following their recruitment by sequencespecific transcription factors. Second, HDAC overexpression could induce hypoacetylation and thus modulate the function of multiple non-histone proteins, including transcription factors and critical cytoplasmic proteins such as Hsp90. HDACs are important regulators of colon cell maturation and transformation, and HDACi are potent inducers of growth arrest, differentiation and apoptosis of colon cancer cells. Treatment of colon cancer cells with HDACi typically induces G0/G1 growth arrest $(33,34)$, and HDACi also induce apoptosis in colon cancer cells $(35,36)$. Based upon numerous pre-clinical findings, several HDAC inhibitors are presently in clinical trials for the treatment of a variety of hematological and solid tumors including colon cancer. One of the inhibitors, SAHA, has already been approved for the treatment of cutaneous T-cell lymphoma (37). In addition, other inhibitors, PXD-101 $(38,39)$, valproic acid (40) and MS-275 (41) are presently being evaluated in clinical trials. For colon cancer, a number of trials have been initiated to determine the efficacy of the combination of SAHA with existing chemotherapeutics including 5FU/ LV and FOLFOX (5FU-leucovorin/oxaliplatin) for treatment of patients with progressive metastatic or unresectable colorectal cancer. Based upon these findings and trials, HDACi are promising in the development of new drugs for the treatment of advanced colon cancer. Thus, immunohistochemical detection of HDAC1 may be a diagnostic resource for the prediction of treatment response to HDACi.

Regarding the molecular mechanism of MTA1 enhancement of tumor metastases, Yoo et al reported that MTA1 enhances the stability and transcriptional activity of hypoxia-induciblefactor- $1 \alpha(\mathrm{HIF}-1 \alpha)$ by recruiting HDAC1 in human breast cancer cells (42). Hypoxia-inducible factor 1 (HIF-1) is the master transcriptional regulator that facilitates adaptation to low oxygen availability (43) and is composed of an HIF-1 $\alpha$ subunit and an HIF-1 $\beta$, also known as the arylhydrocarbon receptor nuclear translocator. The expression of HIF-1 is closely related to tumor growth and metastasis. HIF-1 $\alpha$ was found to be overexpressed in only $29 \%$ of primary breast cancers, but in $69 \%$ of breast cancer metastases (44). A significant positive association was found between increased levels of HIF-1 $\alpha$ protein and vascular endothelial growth factor (VEGF) expression in human colorectal carcinoma (45). HIF-1 $\alpha$ expression correlated with high metastatic risk in a series of unselected patients with invasive breast cancer (46). Yoo et al reported that MTA1 induced the deacetylation of HIF-1 $\alpha$ by increasing the expression of HDAC1, and the positive crosstalk between HIF-1 $\alpha$ and MTA1, mediated by HDAC1 recruitment; this indicates a close connection between MTA1-associated metastasis and HIF-1-induced tumor angiogenesis (42). From these findings, investigation of the usefulness of the detection of HIF-1 $\alpha$ expression to predict treatment response to HDACi is warranted.

In the present study, HDAC1 and MTA1 were demonstrated to be significant prognostic factors in colon cancer. However, particularly for HDAC1, further investigation is needed to ascertain whether immunohistochemical detection of HDAC1 may also be a novel companion diagnostic resource for the prediction of the treatment response to HDACi in patients with colon cancer. Prior to using HDAC1 immunostaining as a routine prognostic biomarker, our findings must be validated in a large prospective study.

\section{Acknowledgements}

This study was supported, in part, by a research grant from the Research Support Foundation of the University of Tokushima and the Cancer Research Project in cooperation with TAIHO Pharmaceutical Co., Ltd. (N.K.).

\section{References}

1. Aaltonen LA and Hamilton SR (eds): World Health Organization Classification of Tumors. Pathology and Genetics of Tumours of the Digestive System. International Agency for Research on Cancer Press, Lyon, 2000.

2. Jemal A, Siegel R, Ward E, et al: Cancer statistics, 2006. CA Cancer J Clin 56: 106-130, 2006.

3. Aggarwal S and Chu E: Current therapies for advanced colorectal cancer. Oncology 19: 589-595, 2005.

4. Yoo CB and Jones PA: Epigenetic therapy of cancer: past, present and future. Nat Rev Drug Discov 5: 37-50, 2006.

5. Minucci S and Pelicci PG: Histone deacetylase inhibitors and the promise of epigenetic (and more) treatments for cancer. Nat Rev Cancer 6: 38-51, 2006.

6. Park JH, Jung Y, Kim TY, et al: Class I histone deacetylaseselective novel synthetic inhibitors potently inhibit human tumor proliferation. Clin Cancer Res 10: 5271-5281, 2004.

7. Toh Y, Yamamoto M, Endo K, et al: Histone H4 acetylation and histone deacetylase 1 expression in esophageal squamous carcinoma. Oncol Rep 10: 333-338, 2003.

8. Song J, Noh JH, Lee JH, et al: Increased expression of histone deacetylase 2 is found in human gastric cancer. APMIS 113: 264-268, 2005.

9. Choi JH, Kown HJ, Yoon BI, et al: Expression profile of histone deacetylase 1 in gastric cancer tissue. Jpn J Cancer Res 92: 1300-1304, 2001.

10. Weichert W, Roske A, Gekeler V, et al: Association of patterns of class I histone deacetylase expression with patient prognosis in gastric cancer: a retrospective analysis. Lancet Oncol 9: 139-148, 2008.

11. Lehmann A, Denkert C, Budczies J, et al: High class I HDAC activity and expression are associated with RelA/p65 activation in pancreatic cancer in vitro and in vivo. BMC Cancer 9: 395, 2009.

12. Patra SK, Patra A and Dahiya R: Histone deacetylase and DNA methyltransferase in human prostate cancer. Biochem Biophys Res Commun 287: 705-713, 2001.

13. Waltregny D, North B, van Mellaert F, et al: Screening of histone deacetylase (HDAC) expression in human prostate cancer reveals distinct class I HDAC profiles between epithelial and stromal cells. Eur J Histochem 48: 273-290, 2004.

14. Krusche CA, Wulfing P, Kersting C, et al: Histone deacetylase-1 and -3 protein expression in human breast cancer: a tissue microarray analysis. Breast Cancer Res Treat 90: 15-23, 2005.

15. Zhang Z, Yamashita H, Toyama T, et al: Quantitation of HDAC1 mRNA expression in invasive carcinoma of the breast. Breast Cancer Res Treat 94: 11-16, 2005.

16. Sasaki H, Moriyama S, Nakashima Y, et al: Histone deacetylase 1 mRNA expression in lung cancer. Lung Cancer 46: 171-178, 2004.

17. Bartling B, Hofmann HS, Boettger T, et al: Comparative application of antibody and gene array for expression profiling in human squamous cell lung carcinoma. Lung Cancer 49: 145-154, 2005. 
18. Cress WD and Seto E: Histone deacetylases, transcriptional control, and cancer. J Cell Physiol 184: 1-16, 2000.

19. Toh Y, Kuninaka S, Endo K, et al: Molecular analysis of a candidate metastasis-associated gene, MTA1: possible interaction with histone deacetylase 1. J Exp Clin Cancer Res 19: 105-111, 2000.

20. Kumar R: Another tie that binds the MTA family to breast cancer. Cell 113: 142-143, 2003.

21. Bowen NJ, Fujita N, Kajita M, et al: Mi-2/NuRD: multiple complexes for many purposes. Biochim Biophys Acta 1677: 52-57, 2004

22. Toh Y, Kuwano H, Mori M, et al: Overexpression of metastasisassociated MTA1 mRNA in invasive oesophageal carcinomas. Br J Cancer 79: 1723-1726, 1999.

23. Toh Y, Ohga T, Endo K, et al: Expression of the metastasisassociated MTA1 protein and its relationship to deacetylation of the histone $\mathrm{H} 4$ in esophageal squamous cell carcinomas. Int J Cancer 110: 362-367, 2004

24. Toh Y, Oki E, Oda S, et al: Overexpression of the MTA1 gene in gastrointestinal carcinomas: correlation with invasion and metastasis. Int J Cancer 74: 459-463, 1997.

25. Hofer MD, Kuefer R, Varambally S, et al: The role of metastasisassociated protein 1 in prostate cancer progression. Cancer Res 64: 825-829, 2004

26. Martin MD, Hilsenbeck SG, Mohsin SK, et al: Breast tumors that overexpress nuclear metastasis-associated 1 (MTA1) protein have high recurrence risks but enhanced responses to systemic therapies. Breast Cancer Res Treat 95: 7-12, 2006.

27. Jang KS, Paik SS, Chung H, et al: MTA1 overexpression correlates significantly with tumor grade and angiogenesis in human breast cancers. Cancer Sci 97: 374-379, 2006.

28. Sasaki H, Moriyama S, Nakashima Y, et al: Expression of the MTA1 mRNA in advanced lung cancer. Lung Cancer 35: $149-154,2002$.

29. Japanese Society for Cancer of the Colon and Rectum: General Rule for Clinical and Pathological Studies on Cancer of the Colon, Rectum and Anus in Japan. 7th edition. Kanehara, 2006.

30. Weichert W, Roske A, Niesporek S, et al: Class I histone deacetylase expression has independent prognostic impact in human colorectal cancer: specific role of class I histone deacetylases in vitro and in vivo. Clin Cancer Res 14: 1669-1677, 2008.

31. Miyake K, Yoshizumi T, Imura S, et al: Expression of hypoxia-inducible factor-1alpha, histone deacetylase 1 , and metastasis-associated protein 1 in pancreatic carcinoma: correlation with poor prognosis with possible regulation. Pancreas 36: $1-9,2008$

32. Mariadason JM: HDACs and HDAC inhibitors in colon cancer. Epigenetics 3: 28-37, 2008.

33. Heerdt BG, Houston MA and Augenlicht LH: Short-chain fatty acid-initiated cell cycle arrest and apoptosis of colonic epithelia cells is linked to mitochondrial function. Cell Growth Differ 8: 523-532, 1997.
34. Schwartz B, Avivi-Green C and Polak-Charcon S: Sodium butyrate induces retinoblastoma protein dephosphorylation, p16 expression and growth arrest of colon cancer cells. Mol Cell Biochem 188: 21-30, 1998.

35. Hague A, Manning AM, Hanlon KA, et al: Sodium butyrate induces apoptosis in human colonic tumor cell lines in a p53-independent pathway: implications for the possible role of dietary fibre in the prevention of large bowel cancer. Int J Cancer 55: 498-505, 1993

36. Heerdt BG, Houston MA and Augenlicht LH: Potentiation by specific short-chain fatty acids of differentiation and apoptosis in human colonic carcinoma cell lines. Cancer Res 54: 3288-3293, 1994.

37. Marks PA and Breslow R: Dimethyl sulfoxide to vorinostat: development of this histone deacetylase inhibitor as an anticancer drug. Nat Biotechnol 25: 84-90, 2007.

38. Marson CM, Mahadevan T, Dines J, et al: Structure-activity relationships of aryloxyalkanoic acid hydroxyamides as potent inhibitors of histone deacetylase. Bioorg Med Chem Lett 17: 136-141, 2007.

39. Bhalla KN: Epigenetic and chromatin modifiers as targeted therapy of hematologic malignancies. J Clin Oncol 23: 3971-3993, 2005.

40. Bolden JE, Peart MJ and Johnstone RW: Anticancer activities of histone deacetylase inhibitors. Nat Rev Drug Discov 5: 769-784, 2006.

41. Rasheed WK, Johnstone RW and Prince HM: Histone deacetylase inhibitors in cancer therapy. Expert Opin Investig Drugs 16: 659-678, 2007.

42. Yoo YG, Kong G and Lee MO: Metastasis-associated protein 1 enhances stability of hypoxia-inducible factor- $1 \alpha$ protein by recruiting histone deacetylase 1. EMBO J 25: 1231-1241, 2006.

43. Semenza GL: Targeting HIF-1 for cancer therapy. Nat Rev Cancer 3: 721-732, 2003.

44. Zhong H, De Marzo AM, Laughner E, et al: Overexpression of hypoxia-inducible factor $1 \alpha$ in common human cancers and their metastases. Cancer Res 59: 5830-5835, 1999.

45. Kuwai T, Kitadai Y, Tanaka S, et al: Expression of hypoxiainducible factor- $1 \alpha$ is associated with tumor vascularization in human colorectal carcinoma. Int J Cancer 105: 176-181, 2003.

46. Dales JP, Garcia S, Meunier-Carpentier S, et al: Overexpression of hypoxia-inducible factor HIF-1 $\alpha$ predicts early relapse in breast cancer: retrospective study in a series of 745 patients. Int J Cancer 116: 734-739, 2005. 